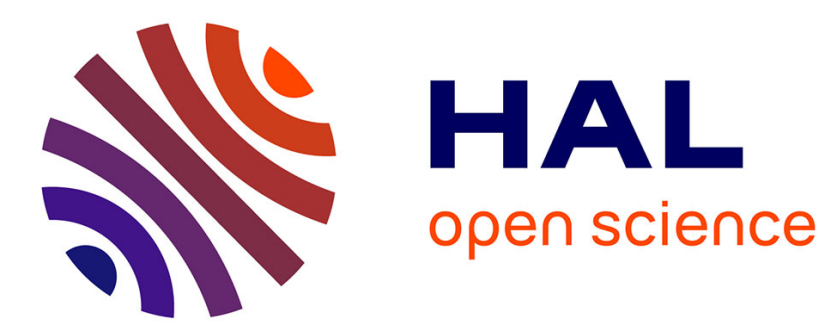

\title{
Phase transition in a domain wall
}

\author{
J. Lajzerowicz, J.J. Niez
}

\section{- To cite this version:}

J. Lajzerowicz, J.J. Niez. Phase transition in a domain wall. Journal de Physique Lettres, 1979, 40

(7), pp.165-169. 10.1051/jphyslet:01979004007016500 . jpa-00231598

\section{HAL Id: jpa-00231598 https://hal.science/jpa-00231598}

Submitted on 1 Jan 1979

HAL is a multi-disciplinary open access archive for the deposit and dissemination of scientific research documents, whether they are published or not. The documents may come from teaching and research institutions in France or abroad, or from public or private research centers.
L'archive ouverte pluridisciplinaire HAL, est destinée au dépôt et à la diffusion de documents scientifiques de niveau recherche, publiés ou non, émanant des établissements d'enseignement et de recherche français ou étrangers, des laboratoires publics ou privés. 


\title{
Phase transition in a domain wall
}

\author{
J. Lajzerowicz $\left(^{*}\right)$ and J. J. Niez $\left(^{* *}\right)$ \\ $\left(^{*}\right)$ Laboratoire de Spectrométrie Physique $\left({ }^{* * *}\right)$ \\ Université Scientifique et Médicale de Grenoble, B.P. 53X, 38041 Grenoble Cedex, France \\ and
}

(**) Département de Recherche Fondamentale,

Centre d'Etudes Nucléaires de Grenoble, B.P. 85X, 38041 Grenoble Cedex, France

(Reçu le 27 décembre 1978, accepté le 16 février 1979)

\begin{abstract}
Résumé. - On étudie une paroi de domaine dans un modèle $X Y$ tridimensionnel en fonction de l'anisotropie $K$ et de la température $T$. On montre que la paroi présente une transition de phase dans le plan $(K, T)$, le paramètre d'ordre étant la chiralité. C'est un système à deux états analogue à un système d'Ising. La transition peut être du second ordre avec modes mous. Dans l'état ordonné, la paroi peut posséder des domaines de chiralités opposées séparés par des lignes singulières.
\end{abstract}

\begin{abstract}
We study a domain wall in a three dimensional $X Y$ model as a function of anisotropy $(K)$ and temperature $(T)$. It is shown that the wall undergoes a phase transition in the $K, T$ plane, the order parameter being the chirality. Thus it is a two state system analogous to an Ising system. The phase transition can be of the second order and exhibits soft modes. In the ordered state the wall can exhibit domains of opposite chirality separated by singular lines.
\end{abstract}

We consider a three dimensional $X Y$ [1] model $(n=2)$ with two dimensional rotators, the rotation axis being the $Z$ direction. The rotators are located at the vertices of a simple cubic lattice, the interaction being between first neighbours. The Hamiltonian of the system can be written :

$$
\begin{aligned}
H & =-J \sum_{i i^{\prime}} \mathbf{S}_{i} \mathbf{S}_{i^{\prime}}-K \sum_{i}\left(S_{i}^{Y}\right)^{2} \\
& =-J \sum_{i i^{\prime}} \cos \left(\theta_{i}-\theta_{i^{\prime}}\right)-K \sum_{i} \cos ^{2} \theta_{i}
\end{aligned}
$$

$\theta_{i}:$ angle between $\mathbf{S}_{i}$ and the $Y$ axis

$i$ : represents the three-dimensional $(p, q, r)$ indices.

When we vary the temperature this system undergoes a bulk phase transition - ferromagnetic ordering which, because of the anisotropy $K$, is of Ising character $(n=1)$.

Let us consider a domain wall perpendicular to the $Z$ direction at $T=0$. When $K$ is large, the domain wall width, $\delta$, is of the order of $a \sqrt{J / K}(a:$ lattice parameter $)$; so this relation loses its meaning when $\delta$ is smaller than $a(J / K \ll 1)[2]$. In this regime $(J / K \ll 1)$, the domain wall is one unit cell spacing, the spins being up for $Z \leqslant 0$ and down for $Z \geqslant 1$ (up refers to the $O y$ direction). Let us call this configuration (I).

The equilibrium conditions for the wall are :

$$
2 J \sum_{i^{\prime}} \sin \left(\theta_{i}-\theta_{i^{\prime}}\right)+K \sin 2 \theta_{i}=0
$$

with the boundary conditions :

$$
\theta(z=-\infty)=0 ; \quad \theta(z=+\infty)=\pi
$$

$\left.{ }^{* * *}\right)$ Laboratoire associé au C.N.R.S. 
These conditions are fulfilled in (I) because $\theta_{i}=0$ or $\pi$. To investigate its stability let us now study small movements around this configuration. The equations of motion are :

$$
I \frac{\mathrm{d}^{2} \theta_{i}}{\mathrm{~d} t^{2}}=-2 J \sum_{i^{\prime}} \sin \left(\theta_{i}-\theta_{i^{\prime}}\right)-K \sin 2 \theta_{i}
$$

( $I$ is the moment of inertia of each rotator).

Let us write $\theta_{i}=\theta_{i}^{0}+\varphi_{i}\left(\theta_{i}^{0}\right.$ equilibrium angles) and linearize (3) :

$$
\begin{gathered}
I \frac{\mathrm{d}^{2} \varphi_{i}}{\mathrm{~d} t^{2}}+2 J \sum_{i^{\prime}}\left(\varphi_{i}-\varphi_{i^{\prime}}\right)+2 K \varphi_{i}=0 \quad \text { when } \quad r \text { or } r^{\prime} \neq 0 \text { or } 1 \\
\left.I \frac{\mathrm{d}^{2} \varphi_{p, q, 0}}{\mathrm{~d} t^{2}}+2 J 4 \varphi_{p, q, 0}-\varphi_{p+1, q, 0}-\varphi_{p-1, q, 0}-\varphi_{p, q+1,0}-\varphi_{p, q-1,0}-\varphi_{p, q,-1}+\varphi_{p, q, 1}\right]+2 K \varphi_{p, q, 0}=0 \\
\left.I \frac{\mathrm{d}^{2} \varphi_{p, q, 1}}{\mathrm{~d} t^{2}}+2 J 4 \varphi_{p, q, 1}-\varphi_{p+1, q, 1}-\varphi_{p-1, q, 1}-\varphi_{p, q+1,1}-\varphi_{p, q-1,1}-\varphi_{p, q, 2}+\varphi_{p, q, 0}\right]+2 K \varphi_{p, q, 1}=0
\end{gathered}
$$

obviously the solutions have the form

$$
\varphi_{K \|}(p, q, r)=\exp \left[i\left(\omega_{K \|} t-\mathbf{K}_{\|} \mathbf{R}_{(p, q)}\right)\right] \psi_{K \|}(r) .
$$

For a given value of $\mathbf{K}_{\|}$, we have a full spectrum consisting of a localized mode (in the $Z$ direction), $\omega_{\mathrm{L}}$, and a branch of delocalized modes $\omega_{K \|}\left(K_{\perp}\right)$ :

$$
\omega_{\mathrm{L}}^{2}=\omega_{\mathrm{L}}^{02}+\frac{4 J}{I}\left[2-\cos K_{X} a-\cos K_{Y} a\right]
$$

with

$$
\omega_{\mathrm{L}}^{02}=\frac{2 J}{I}\left(\frac{K}{J}-\frac{4}{3}\right)
$$

and

$$
\omega_{K \|}^{2}\left(K_{\perp}\right)=\frac{2 K+4 J\left(1-\cos K_{Z} a\right)}{I}+\frac{4 J}{I}\left[2-\cos K_{X} a-\cos K_{Y} a\right]
$$

The expression (5) has meaning only if $K / J-4 / 3$ is positive. So the condition of stability of configuration (I) is $K / J \geqslant 4 / 3[3,4,5]$. If we look at $\omega_{\mathrm{L}}^{02}$ as a function of $K / J$, we have a typical soft mode behaviour, the eigenvector corresponding to this mode near the threshold value $(K / J)_{c}=4 / 3$ being :

$$
\begin{array}{ll}
\varphi(p, q, r)=\left(\frac{1}{3}\right)^{-r} A & r \leqslant 0 \\
\varphi(p, q, r)=-\left(\frac{1}{3}\right)^{r-1} A & r \geqslant 1
\end{array}
$$

with a typical width of the order of $2 a$.

Just below the threshold, the solution of (2) in the third order in $\theta$ gives

$$
\begin{array}{ll}
\theta(p, q, r)=\varepsilon\left(\frac{1}{3}\right)^{-r} \frac{9}{\sqrt{32}}\left(\frac{4}{3}-\frac{K}{J}\right)^{1 / 2}, \quad(\varepsilon= \pm 1), & r \leqslant 0 \\
\theta(p, q, r)=\pi-\theta(p, q, 1-r) & r \geqslant 1
\end{array}
$$


which is a narrow domain wall with a helicoidal structure (Bloch character). This is another stable configuration (II) which was prefigured by the eigenvector (7). If we look at the domain wall as a function of $K / J$, we see a phase transition with a soft mode. The order parameter is the amplitude of the localized modes given by the value of $\theta(r=0)$ which varies typically as $\left(K / J-(K / J)_{c}\right)^{1 / 2}$. This value of the critical exponent is due to the molecular field approximation (we have neglected quantum fluctuations).

1. Thermodynamic behaviour. - We want to study this system as a function of $T$. From an heuristic point of view, we know that for a given value of $T$, near $T_{\mathrm{c}}, K$ is a relevant quantity and will impose an Ising behaviour for the system. Thus just below $T_{\mathrm{c}}$, the domain wall must have an Ising character (without chirality). On the other hand at low temperature we know that the domain wall will have a Bloch character, so we must have a phase transition within the domain wall as a function of $T$.

To study this problem, we will look at two limiting regimes $T \simeq 0$ and $K \simeq 0$.

$1.1 T$ NEAR $0, \frac{K}{J}=\frac{4}{3}-\varepsilon$. We can associate with each line of spins, $n$, perpendicular to the wall a quantity $\varepsilon_{n}\left(\varepsilon_{n}= \pm 1\right)$ which characterizes the chirality of the line. The " $\theta$ » angles of the line are given by (8). The interaction energy between two neighbouring lines $n$ and $n^{\prime}$ can be written, knowing Hamiltonian (1), in the form :

$$
\tilde{H}=\tilde{J} \varepsilon_{n} \varepsilon_{n^{\prime}}
$$

where

$$
\tilde{J}=\frac{9^{3}}{32} J\left(\frac{4}{3}-\frac{K}{J}\right)
$$

this Hamiltonian (9) exhibits clearly the features of a two-dimensional Ising model with a critical temperature

$$
k T^{*}=\frac{\tilde{J}}{\operatorname{Arctanh}(\sqrt{2}-1)}
$$

1.2 $K$ NEAR 0 . - We know that the domain wall width varies as $a \sqrt{J / K}$, so we will go to a continuum description. Let us call $X$ and $Y$ the magnetization components. The three-dimensional Ginzburg-Landau Free Energy corresponding to the Hamiltonian (1) is of the form :

$$
\begin{aligned}
F_{\mathrm{L}}=J a^{2}\left[(\operatorname{grad} X)^{2}\right. & \left.+(\operatorname{grad} Y)^{2}\right]- \\
& -(6 J-k T+K)\left(X^{2}+Y^{2}\right) \\
& +K X^{2}+B\left(X^{2}+Y^{2}\right)^{2} .
\end{aligned}
$$

The uniform solution corresponds to a paramagnetic case for $T>T_{\mathrm{c}}\left(T_{\mathrm{c}}=\frac{6 J+K}{k}\right)$ and a ferro- magnetic case for $T<T_{\mathrm{c}}$. We look at a domain wall perpendicular to the $Z$ direction in such a way that the « $\operatorname{grad} »$ terms are $\partial / \partial z$.

The Euler-Lagrange equations associated with our problem have been solved by Sarker et al. [6]. We have two regimes :

i) $k\left(T_{\mathrm{c}}-T\right)<2 K$ the domain wall exhibits an Ising character

$$
\left\{\begin{array}{l}
X=0 \\
Y= \pm \sqrt{\frac{k\left(T_{\mathrm{c}}-T\right)}{2 B}} \tanh \left[\frac{1}{2} \frac{k\left(T_{\mathrm{c}}-T\right)}{J}\right]^{1 / 2} \frac{z}{a}
\end{array}\right.
$$

ii) $k\left(T_{\mathrm{c}}-T\right)>2 \mathrm{~K}$ the domain wall exhibits a Bloch character (with definite chirality)

$$
\left\{\begin{array}{l}
X=\mp \sqrt{\frac{k\left(T_{\mathrm{c}}-T\right)-2 K}{2 B}} \operatorname{sech} \frac{z}{\lambda} \\
Y= \pm \sqrt{\frac{k\left(T_{\mathrm{c}}-T\right)}{2 B}} \tanh \frac{z}{\lambda}
\end{array}\right.
$$

where $\lambda$ is the characteristic width of a Bloch wall $(\lambda=a \sqrt{J / K})$.

The transition temperature is given by

$$
k T^{*}=k T_{\mathrm{c}}-2 K .
$$

It is easy to show, that if we take a Ginzburg-Landau time dependent expansion, we have also soft modes connected to this transition [7]. All the critical exponents are of mean field character as a result of the approximations. It is obvious that, in this case, we have the exponents of a two dimensional Ising model except at $T=0$ where we can conjecture that the critical exponents will be those of a three-dimensional Ising model.

The results are summarized in figure 1 .

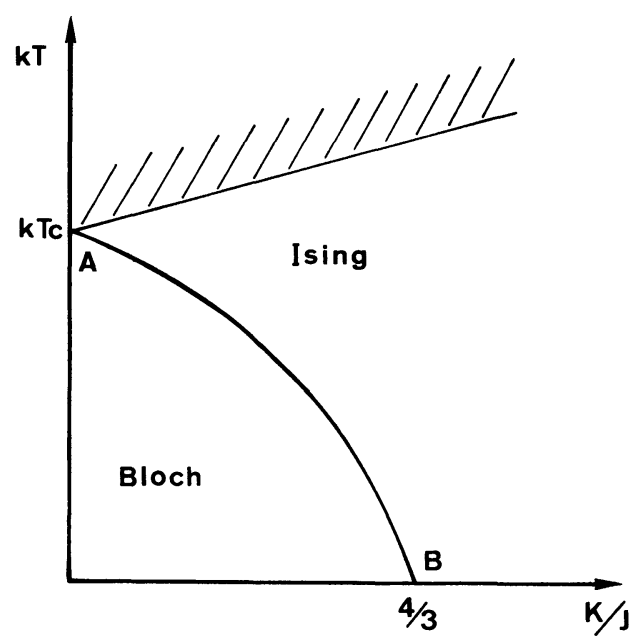

Fig. 1. - The AB line is the transition line between a domain wall of Ising type and a domain wall of Bloch type (chiral). The shaded region corresponds to the bulk paramagnetic state. 
2. Other type of ordered state. - We will show in one definite example that the structure of the ordered domain wall can be more complex. We now limit the discussion to $T=0$. The frequency (5) of the localized mode associated with configuration (I) depends on $\omega_{\mathrm{L}}^{0}$ on one hand and on the dispersion relations of the excitations of spin wave type in the $(X, Y)$ plane on the other hand. So, if one introduces in our model an antiferromagnetic exchange between next nearest neighbours in the $X$ and $Y$ directions, one modifies the dispersion relations in these directions in such a way that $\omega_{\mathrm{L}}$ can be zero when $\omega_{\mathrm{L}}^{0}$ is still nonzero. Thus, now, we are going to consider a Hamiltonian of the same kind as that used by Redner and Stanley [8] :

$$
H=-2 J \sum_{\langle i j\rangle} \mathbf{S}_{i} \mathbf{S}_{j}+2 J_{2} \sum_{\langle i j\rangle}^{X Y} \mathbf{S}_{i} \mathbf{S}_{j}-K \sum_{i}\left(S_{i}^{Y}\right)^{2}
$$

where the second term represents an exchange between next nearest neighbours in the $(X, Y)$ plane.

In this case, the frequency of the localized mode becomes :

$$
\begin{aligned}
\omega_{\mathrm{L}}^{2}\left(K_{X}, K_{Y}\right) & =\omega_{\mathrm{L}}^{02}+ \\
+ & \frac{4 J}{I}\left\{\left[2-\cos K_{X} a-\cos K_{Y} a\right]\right. \\
- & \left.\frac{J_{2}}{J}\left[2-\cos 2 K_{X} a-\cos 2 K_{Y} a\right]\right\}
\end{aligned}
$$

and we have two regimes :

- if $\frac{4 J_{2}}{J}<1, \omega_{\mathrm{L}}=0$ for $\frac{K}{J}=\frac{4}{3}\left(\omega_{\mathrm{L}}^{0}=0\right)$ the soft mode has a uniform structure in the $(X, Y)$ plane and the wall which appears for $\frac{K}{J}=\frac{4}{3}-\varepsilon$ is of uniform Bloch type;

- if $\frac{4 J_{2}}{J}>1, \omega_{\mathrm{L}}\left(K_{X}, K_{Y}\right)$ is minimum for

$$
\left(K_{X}, K_{Y}\right)=\left(q_{X}, q_{Y}\right)
$$

with $\cos q_{Y} a=\cos q_{X} a=\frac{J}{4 J_{2}}$. It is easy to show that $\omega_{\mathrm{L}}\left(q_{X}, q_{\mathrm{X}}\right)$ is zero when

$$
\frac{K}{J}=\frac{4}{3}+\frac{J}{2 J_{2}}\left(1-\frac{4 J_{2}}{J}\right)^{2}
$$

the soft mode exhibiting a modulated structure associated with the wave vectors $q_{X}$ and $q_{Y}$ in the $X$ and $Y$ directions. Consequently the wall which appears for $\frac{K}{J}$ just below $\frac{4}{3}+\frac{J}{2 J_{2}}\left(1-\frac{4 J_{2}}{J}\right)^{2}$ has a modulated structure. In such a situation if one has a bulk phase transition from a ferromagnet to a helimagnet (III $\rightarrow$ IV), as the domain wall of the ferromagnet has already an helical character, the nucleation of the bulk helicoidal state will occur in the domain wall (the width of the wall diverges on line $c$-). Such an hypothesis has already been made to interpret experimental results [9].

These results show that there must also exist a line, in the $\left(\frac{K}{J}, \frac{4 J_{2}}{J}\right)$ plane, giving the transition between Bloch wall and modulated Bloch wall. The results summarized in figure 2 indicate the presence of a Lifshitz point [10].

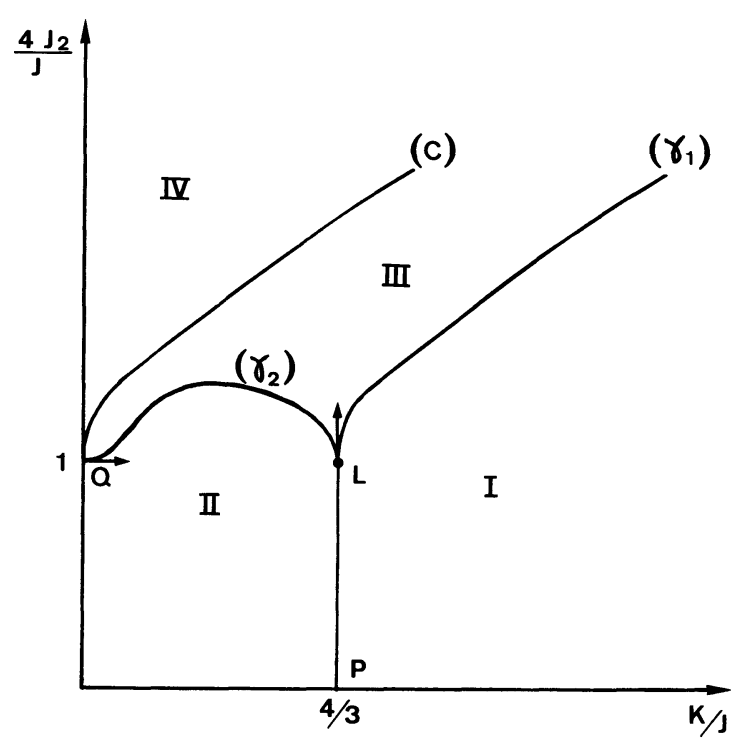

Fig. 2. - Phase Diagram at $T=0$. The region $\mathrm{I}$ corresponds to configuration (I); The region II corresponds to uniform Bloch wall ; The region III corresponds to modulated Bloch wall; The region IV corresponds to a bulk helicoidal type of state; $\mathbf{L}$ is a Lifshitz point ; $\left(\gamma_{1}\right)$ is given by $(13) ;\left(\gamma_{2}\right)$ is a numerically calculated ; (C) characterizes the transition between uniform and modulated structures in the bulk and is parallel to $\left(\gamma_{1}\right)$. One can show that LP, $\left(\gamma_{1}\right),\left(\gamma_{2}\right)$ have a vertical tangent at L. At $Q,(C)$ has a vertical tangent, and $\gamma_{2}$ a horizontal one.

3. Conclusion. - We have shown that a ferromagnetic domain wall can undergo a two-dimensional phase transition in space with parameters $\left(J, J_{2}\right.$, $K, T)$. The broken symmetry is the chiral symmetry (right-left). In the ordered state, singularities associated with the broken symmetry are Bloch lines. This transition is associated with the fact that the dimensionality, $n$, of the order parameter is greater than one and is different from the roughening transition [11]. The bulk phase transition locks one of the components of the order parameter; the transition then occurs in the space of the $(n-1)$ other components. This shows that the singularities associated with ordered media [12] can change their nature in a cooperative way. Thus their degree of universality is less than that of the bulk transition; that is to say they are more sensitive to the structure of the Hamiltonian. We have presented another example of a phase transition in two dimensions [13].

Acknowledgments. - Thanks are due to Dr. P. Averbuch, P. Nozières and G. Toulouse for several discussions concerning this paper, and to Dr. P. Baruchel for indicating to us reference [10]. 


\section{Footnotes and references}

[1] This model can be used for a Heisenberg system with strong planar anisotropy in the $X Y$ plane induced by magnetostatic interactions. The usual localized spin waves are degenerated in the absence of dipolar interactions. In fact this interaction is a relevant one; it splits the degeneracy and so we consider only (near the transition) the lowest branch. Physically the spins cannot get out of the $X Y$ plane because of the pole field. This amounts to an associated moment of inertia $I \sim \frac{\hbar^{2} V^{-1}}{M_{\mathrm{s}}^{2}}$ where $V$ is the volume of the unit cell (one magnetic moment by unit cell) and $M_{s}$ the spontaneous magnetization.

[2] This situation can be found in systems like $\mathrm{Dy}_{3} \mathrm{Al}_{2}$ etc...

[3] ZiJlstra, H., IEEE Trans. Magn. 6 (1970) 179.

[4] Barbara, B., Becle, C., Lemaire, R. and Paccard, D., C.R. Hebd. Sean. Acad. Sci. 271 (1970) 880.
[5] Niez, J. J., J. Phys. C. 9 (1976) 2933.

[6] Sarker, S., Trullinger, S. E., Bishop, A. R., Phys. Lett. A 59 (1976) 255

[7] If we include a damping term instead of soft mode we will have a spectrum of relaxation times with $\frac{1}{\tau_{0}} \rightarrow 0$ at the transition point.

[8] Redner, S. and Stanley, H. E., Phys. Rev. 16 (1977) 4901.

[9] Palmer, S. B., J. Phys. F 5 (1975) 2370.

[10] Hornkeich, R. M., Luban, M. and Shtrikman, S., Phys. Rev. Lett. 35 (1975) 1678.

[11] Leamy, H. J., Gilmer, G. H., JACKSON, K. A., Surface Physics of Materials (Blakely ed., Academic, NY) 1 (1975) 121188.

[12] Toulouse, G. and Kléman, M., J. Physique Lett. 37 (1976) 149.

[13] Mctague, J. P. and Nielsen, M., Phys. Rev. Lett. 37 (1976) 596. 